\title{
EL EXTREMO AUSTRAL SUDAMERICANO. OCUPACIÓN Y RELACIONES DE LOS TERRITORIOS ARGENTINOS Y CHILENOS, 1880-1920
}

\author{
Por \\ Elsa Mabel Barbería*
}

\begin{abstract}
RESUMEN
Este artículo se centra en el estudio del poblamiento de los territorios argentinos fronterizos con Chile, en particular, el de Santa Cruz y Tierra del Fuego. Dentro del marco de la expansión capitalista, se describen las diversas etapas por las que este proceso demográfico pasó hasta el poblamiento definitivo de la zona en el siglo XIX. Asimismo, se analizan las actividades económicas que estimularon el desarrollo de la región, en especial la producción ovina, y el rol que los grandes empresarios desempeñaron en su conformación y funcionamiento. Para finalizar, se exponen los factores tanto nacionales e internacionales que dieron como resultado la desintegración de la zona.
\end{abstract}

\begin{abstract}
This article revolves about the study of the population fo the Argentina-Chile frontier territories, particularly that of Santa Cruz and Tierra del Fuego. Within the framework of capitalist expansion, a description is given of the various stages through which this demographic process passed, leading to the definitive poputation of the zone in the neneteenth century. An analysis is given as well of the economic activities, which stimulated the development of the region, especially the raising of sheep, and the role that the great impresarios played in its formation and function. The article ends by showing national and international factors which led to the desintegration of the area.
\end{abstract}

\section{INTRODUCCIÓN}

A mediados del siglo XIX se inicia el poblamiento definitivo del extremo austral sudamericano. El mismo se inscribe en el marco del proceso de expansión del capitalismo hacia las regiones periféricas, en este caso para proveerse de lana para la creciente industria textil.

* Investigadora del Consejo Nacional de Investigaciones Científicas y Técnicas y de la Universidad Federal de la Patagonia Austral, Argentina. 
La producción ovina en la región se origina en las Islas Malvinas, de allí se expande al sur de Chile y luego a los territorios argentinos de Santa Cruz y Tierra del Fuego (ver figura 1).

Durante el periodo que se extiende entre 1880 y 1920 , aproximadamente, se conjugan condiciones excepcionales que favorecen el poblamiento del extremo austral sudamericano. En esta etapa los territorios que integran el área conforman una región con autarquía de sus respectivas capitales nacionales, especializada en la producción lanera con destino al mercado europeo.

Este artículo se centra en el análisis de esa región, especialmente en el poblamiento del sector argentino. De acuerdo con la ley núm. 1532, del año 1884, la hasta entonces denominada Patagonia, se dividía en cínco territorios. Los dos más australes, y a los que nos referimos particularmente son el de Santa Cruz, que limita al norte con el de Chubut y al sur con Chile, y cuya capital fue instalada en Puerto Santa Cruz, trasladándose a Río Gallegos en 1887; y el de Tierra de Fuego, que comprende la parte este de la isla del mismo nombre, con capital en Ushuaia.

En primer lugar se presentan los primeros intentos poblacionales; en segundo lugar el poblamiento definitivo de la región, iniciado en Malvinas y sur de Chile; en tercer lugar, la ocupación de los territorios argetinos; y, por último, la formación y funcionamiento de la región, el rol de los grandes empresarios y la desintegración de la zona.

\section{LOS PRIMEROS INTENTOS POBLACIONALES}

Luego del protagonismo que tuvo el extremo austral sudamericano a principios del siglo $\mathrm{XVI}$, cuando afanosamente se buscaba el paso que comunicara ambos océanos, sobrevino una etapa que si bien reconoció la presencia de expediciones de diferentes estados europeos, no hubo un interés concreto de ocupación.

Después del descubrimiento del estrecho que comunicaba el océano Atlántico con el Pacífico, fueron escasos los intentos de poblamiento en la región. En 1520, la corona española encomienda a Pedro Sarmiento de Gamboa la fundación de dos asentamientos que posibilitaran su defensa. Estos fueron la "Ciudad Nombre de Jesús" y "Real Felipe", ambas nacidas el 11 de febrero de 1584; la primera de ellas y ubicada en el actual territorio argentino y la segunda en el chileno.

Pero ambos fueron tan sólo símbolos de soberanía, ya que las funciones de defensa que les fueran acordadas no se cumplieron, desde el momento en que se deja a los pobladores a su propia suerte; a los pocos años ningún español sobrevivía en la región. 


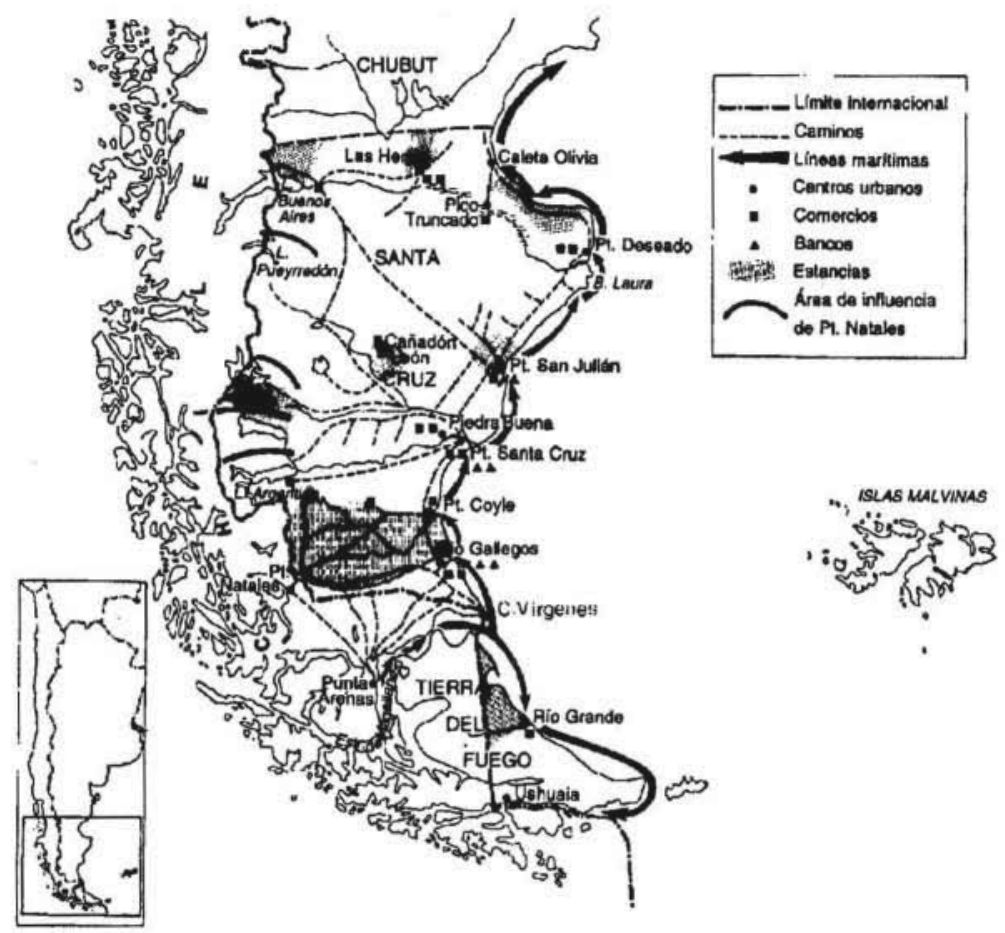

Figura 1. Expansión de capitales procedentes de Chile en territorio argentino.

FUENTE: Elaboración propia con datos de Martinic Beros y Correa Falcón. 
Posteriormente, en España renació el ímpetu poblador cuando se intensificaron las expediciones de otros países, para 1780 surgió otra población en la región la "Nueva Colonia de Floridablanca", ubicada en las cercanías de la bahía de San Julián - actual territorio argentino-, la que logró mantenerse casi cuatro años, debido al contacto que sus pobladores entablaron con los tehuelches.

En 1789 se fundo la "Real Compañía Marítima" en Puerto Deseado, a consecuencia del aumento de loberos y balleneros británicos, comunidad que contó, a diferencia de las otras, con una actividad económica que permitió vivir a sus babitantes sin ayuda de la corona. Esa actividad consistra en pesca, caza marina, salazón de cueros y obtención de aceite de anfibios. Pero la mala administración y la falta de apoyo la condujeron al fracaso, debiendo sus pobladores recurrir, nuevamente, a los hospitalarios tehuelches, con los que negociaron plumas de avestruz y pieles de guanaco.

Una vez lograda la independencia de la corona española y conformados los estados de Argentina y Chile, pasaron también muchos años hasta que los gobiernos nacionales pusieran sus ojos en la Patagonia Austral, que se convertiría también en un problema limítrofe entre ambos.

Chile fue quien inició el poblamiento de estos lejanos territorios; en 1843 funda Fuerte Bulnes, a los pocos años se decide su traslado más al norte, a un sitio conocido como Punta Arenosa, de donde deriva Punta Arenas. Al comienzo fue tan sólo un presidio, destruido en 1852 por una sublevación, pero a partir de 1867 renace y cobra impulso mediante una política decidida de atracción de pobladores y desarrollo económico en esta zona.

La importancia que cobró el Estrecho de Magallanes como vía del comercio internacional, convirtió a Punta Arenas en sitio obligado de recalada para el aprovisionamiento de leña, agua, carne y hortalizas. En 1865, se inaugura la navegación regular con Europa por medio del servicio de vapores de la Pacific Steam Navigation que unía Punta Arenas con Valparaiso y Liverpool, a la que seguirán varias compañías más.

En la década de los setenta del siglo pasado, se intensifica la llegada de inmigrantes y comienzan las primeras actividades productivas que son: a) el aprovechamiento de las arenas auriferas en distintos puntos de la región; $b$ ) la recuperación de cargamentos de naufragios (denominados "raques") - muy comunes en la zona, provocados por los fuertes vientos y el gran oleaje-, la mercadería obtenida se vendía en los comercios; debido al auge que tomaron los "raques" se oficializo su práctica mediante la entrega de concesiones otorgadas por la autoridad marítima; y $c$ ) la caza de lobos marinos, importante rubro de exportación. A estas actividades 
mencionadas seguirán, la extracción de carbón, destinada a las embarcaciones; y la explotación forestal, que creció más tarde con el avance de la producción ganadera (Martinic, 1976 y 1972).

En lo que se refiere al territorio argentino, en Santa Cruz, el primer hombre blanco que se instaló fue el marino Luis Piedra Buena en la isla Pavón, en 1859, quien se dedicó a la caza de anfibios, a la que sumó la extracción de sal y la comercialización de plumas de avestruz y pieles de guanaco, fruto del trueque con los indígenas, a quienes provee de otras mercaderías. A Piedra Buena lo siguieron sus parientes y compañeros de navegación, conformándose así un incipiente asentamiento. Por su parte, el gobierno nacional designa un delegado, pero el alejamiento de Piedra Buena, en 1878, y el establecimiento de una Subdelegación Marítima en Puerto Santa Cruz, producen el fin del mismo.

Otro intento se produjo en 1872, con la instalación de un industrial francés, Ernesto Rouquard, en Puerto Santa Cruz. Él se dedico a la pesca, salazón de anfibios y obtención de aceite y harina; solicitó también terrenos para destinarlos a la colonización. Sin embargo, el fracaso de la empresa, las desdichas personales, la superposición de terrenos con una concesión posterior y las incursiones de los chilenos, lo obligaron a abandonar el lugar.

El tercer intento, que si bien se originó en 1876, cobró vigor después de 1880, fue el descubrimiento de arenas auríferas en Cabo Vírgenes, que atrajo a buscadores llegados de Punta Arenas y de Buenos Aires, y que dio lugar a la llamada "fiebre del oro".

En 1886, el gobiemo nacional decreta la instalación de un pueblo y una Ayudantía Marítima. Algunas personas sostienen que en el mejor momento llegó a concentrar a doscientos buscadores. Pero todo fue muy efímero.

Si bien la explotación del oro no constituyó una actividad importante en Santa Cruz, como lo fue en Chile, sí generó acumulación de capital invertido en la ganadería ovina, ya que algunos buscadores compraron ganado y arrendaron tierras,

Por su parte, en la Tierra del Fuego en Argentina, se había establecido una misión anglicana en Ushuaia; y a partir de 1886 Julio Popper, el buscador de oro más conocido de la región debido a los buenos resultados que obtuvo, en especial de su yacimiento más importante denominado "El Páramo", como por sus excentricidades, dio mucho de que hablar a la prensa de Buenos Aires y logró convertirse en un personaje de leyenda. LLegó a acuñar monedas, realizó diversas exploraciones por la isla, y conformó un grupo para vigilar sus yacimientos, que según los periodistas se transformo en "un ejército propio". 


\section{EL POBLAMIENTO DEFINITIVO. LA GANADERÍA OVINA}

La historia de la ganadería en la región se inició en las Malvinas. Después de ser tomadas por los ingleses, se instalaron algunos pobladores que probaron suerte con los bovinos. Los empresarios pagaban una cuota por el arrendamiento de las tierras y un porcentaje sobre la producción de cueros.

Los cueros eran exportados en veleros, que venían con carga para Buenos Aires y Montevideo, de donde se dirigían, casi en lastre, a las Malvinas. Al principio llevaban únicamente lo necesario para la vida de los escasos habitantes de estas islas, hasta que en 1859, empezaron a llevar ovejas desde Buenos Aires y especialmente de Montevideo (Morrison, 1917:54-55).

La Falklands Islands Company, empresa londinense, inició la ganadería ovina en Malvinas adquiriendo una concesión de 500000 hectáreas, monopolizando la comercialización lanera de los pobladores llegados a partir de 1867 de Escocia, Gran Bretaña, Australia y Nueva Zelandia.

La característica de la distribución de la tierra en Malvinas, fue la concentración en manos de compañías que luego sumaron grandes extensiones en el sur de Chile y en los territorios argentinos. En cuanto se refiere a la ganadería ovina, contribuyó con una raza adaptada a la región austral, conocida como Malvinera - producto de la cruza de la oveja criolla con otras de origen inglés (Cheviot, Romney Marsh, Lincoln y Leicester)-, que luego se difundió en toda la zona; con un modelo empresarial de producción y con un grupo importante de capataces y ovejeros - especialmente escoceses - formados en las duras condiciones regionales, que más tarde fueron administradores o estancieros en Argentina y Chile. En 1877 nace el primer establecimiento ganadero en el sur de Chile; y en la década de los ochenta del siglo pasado avanza sostenidamente la frontera ovina. En los inicios las tierras fueron entregadas en arrendamiento y, a principio de siglo, fueron ofrecidas en venta, mediante remate público. Este método que no concede ningún derecho a los ocupantes originales, permite la concentración desmedida; es así que se produce el predominio del latifundio en manos de muy pocas sociedades anónimas. Los arrendatarios que quedan sin tierras emigraron a los territorios argentinos.

El historiador chileno, Mateo Martinic Beros, señala que en 1892 comienza la "edad de oro" del sur de Chile, caracterizada por el incremento de las actividades productivas iniciadas en la década de los ochenta, a las que se suman la explotación del cobre y los astilleros y talleres de reparación naval, surgidos por la importancia que cobra el cabotaje regional, a consecuencia de la expansión de la producción ovina. 


\section{LA OCUPACIÓN DE LOS TERRITORIOS ARGENTINOS}

El proceso de expansión del capitalismo, motivado por el interés de incorporar nuevos mercados, de asegurar materias primas a bajo costo y de obtener mayores beneficios en las inversiones, $\mathrm{y}$ el rol asignado a la Argentina - exportadora de productos agrícola-ganaderos - condujo a la decisión de integrar nuevas tierras y redefinir el uso de las ya incorporadas, para aumentar la producción; y al desplazamiento del ovino a las áreas más alejadas del puerto de Buenos Aires, campos que convenía destinar al vacuno y a los cereales.

En consecuencia, la Patagonia se puebla como parte de la política de conquista e incorporación de los territorios nacionales. Tampoco hay que desconocer en esta decisión, la necesidad de sentar soberanía, especialmente por los reclamos de Chile, en relación con la zona sur de Santa Cruz, que llega incluso a establecer una Capitanía Marítima.

La firma del Tratado Internacional de Límites, firmado por ambos países en 1881, puso fin a las controversias, al menos por un tiempo prolongado. El abrazo entre el presidente chileno, Federico Errázuriz, y el argentino Julio Argentino Roca, concretado en la región en 1899, avalaron el acuerdo e impulsaron la ocupación de los territorios argentinos fomentando la instalación de los pobladores del sur chileno (Lafuente, 1981).

Pero la decisión nacional de incorporar el sur se combina con el interés de los capitalistas de la región -Malvinas y sur de Chile- por ampliar el ámbito de sus inversiones en estos territorios, aprovechando la coyuntura internacional favorable para la producción lanera. Son las sociedades anónimas, que complementaban la explotación de enormes extensiones de terrenos con actividades relacionadas con el comercio, las finanzas y el transporte, las que propician la ocupación.

También abundaban otros pobladores con capital suficiente para iniciar un establecimiento propio, formado con base en actividades comerciales, caza de anfibios o lavaderos de arenas auriferas; empleados jerarquizados de compañías de transporte marítimo; $y$, por último, estaban aquéllos que habían logrado reunir un modesto capital, por lo general capataces y ovejeros de Malvinas, que apoyados en amigos o en créditos bancarios 0 de sociedades anónimas intentaron ascender social y económicamente, dadas las inmejorables condiciones para iniciarse en la producción lanera.

Este proceso de poblamiento, centrado en la ocupación de la tierra y en su uso para la ganadería ovina, cubrirá más de medio siglo, ya que recién en la década del cuarenta del presente siglo otra actividad dinamizará la región, ésta es la extracción de energéticos — gas, petróleo y carbón-que generará otra etapa del proceso. 
Ahora bien, dentro del periodo se distingue un primer momento - entre 1880 y 1920 - que podemos denominar "auge del ovino", que se caracteriza por la creciente demanda de lana por parte de algunos países europeos y el constante ascenso del precio. En los primeros años se pagaba $\$ 8$ por $10 \mathrm{~kg}$ llegando a $\$ 14$ en 1914 y alcanzando el punto máximo durante la guerra (\$30), a partir de 1918 comienza a descender, igualando el precio original (Sarobe, 1935).

La importancia del Estrecho de Magallanes en el comercio mundial, que además de permitir la comunicación directa y regular con el mercado europeo, utilizando los buques de las ocho compañías de navegación internacionales con sede en Punta Arenas, incentivó el arribo de inmigrantes, atraídos por la posibilidad de acceso a la tierra y por las actividades generadas por la ganadería ovina.

\section{La política nacional implementada}

Lograda la unificación del país, se inicia la etapa de organización política y modemización de la economía; siendo una de sus principales medidas la incorporación de los territorios nacionales, mediante la exploración de las zonas desconocidas, la conquista del desierto, y el dictado de la Iegislación requerida para su ocupación.

La conquista no fue necesaria, el indígena no fue un obstáculo, sino que jugó un rol destacado en los intereses soberanos de Argentina y Chile en la region; ambos se esforzaron por conseguir su reconocimiento mediante halagos, regalos, títulos militares, alcohol; y los particulares negociaron con ellos para obtener plumas y pieles, que colocaban en los mercados europeos. En consecuencia, al momento de introducir el ovino, el tehuelche ya estaba de alguna manera incorporado al sistema, habiéndose iniciado el proceso de destrucción de estos grupos indígenas, por el contagio de enfermedades y el alcohol.

La política nacional sobre los territorios australes puede calificarse de excesivamente débil y con total ausencia de apoyo real a los pobladores. La participación estatal se circunscribió a cubrir funciones administrativas y de seguridad. Las primeras a cargo del gobemador que tuvo escasas atribuciones y fue simplemente un delegado nacional. En cuanto a las funciones de seguridad, éstas se circunscribían solamente a la defensa de las costas por medio del establecimiento de subdelegaciones marítimas.

En relación con la política aduanera, el estado adoptó una actitud más efectiva, impelido por las circunstancias, ya que Chile había eliminado la aduana en 1868. Los pobladores y los gobernadores exigían la adopción de la misma medida para Santa Cruz y Tierra del Fuego. 
Edelmiro Mayer, gobernador de Santa Cruz, escribía en 1893:

...estas comarcas son absolutamente tributarias de Chile y de los países extranjeros. Todo lo que allí se consume es importado y se paga a oro... En cuanto a las exportaciones, los puertos argentinos no tienen casi renta debido a los altos derechos de aduana. En el territorio chileno los puertos son libres y de este modo pueden exportarse lo que en esos territorios se recóge. Para darle vitalidad a esas comarcas sería necesario que el Congreso estableciera en el Sud la absoluta libertad de puertos, pues de otro modo la República Argentina perderá todo lo que Chile gana... (El diario, 1 de junio de 1893 en Isola, s/f).

La eliminación de la aduana —en 1899-obró como incentivo, ya que además de permitir la obtención de mayores beneficios a las empresas por la ausencia de impuestos a la exportación de la producción, disminuía notablemente los costos de inversión y funcionamiento, ya que todo to requerido se compraba directamente en Europa.

Los hacendados enviaban dos veces al año sus pedidos seleccionados de los catálogos de grandes casas comerciales, hasta fines de siglo en que fueron sustituidos por las firmas dedicadas a la importación y exportación, que instalaron sucursales en todos los puertos de la Patagonia Austral.

Además de la presencia del gobernador, policía y prefectos, la distribución de la tierra era el único lazo que unía a los pobladores con el gobierno nacional, ya que los contratos de arrendamiento, compra o permisos de ocupación se gestionaban ante la Dirección Nacional de Tierras y Colonias, con sede en la capital federal.

\section{La ocupación de la tierra en Santa Cruz y Tierra del Fuego ${ }^{1}$}

La política adoptada para la transferencia de terrenos en los territorios de Santa Cruz y Tierra del Fuego a los particulares fue la misma seguida para

1 En relación con la distribución de la tierra se siguen los resultados del trabajo Barbería, Elsa Mabel, 'El proceso de ocupación de la tierra en el territorio de Santa Cruz, 1880-1920" (1994), que encara un análisis minucioso de las diferentes variables intervinientes considerando cada caso particular. Para Tierra del Fuego no existe un estudio de este tipo, si bien las características son similares, ya que responden a una misma política. Las dimensiones y la variedad de terrenos de Santa Cruz presentan mayores diferencias en los ocupantes.

Las categorías de establecimientos ganaderos a las que se hace referencia fueron determinadas en función del análisis interrelacionado de las siguientes variables: superficie, calidad de los terrenos, ubicación, forma de tenencia, momento de la ocupación y características de los ocupantes.

Se fijaron cuatro categorias: 1) latifundios (más de 75000 hectáreas); 2) grandes establecimientos (entre 30000 y 75000 hectáreas); 3) medianos establecimientos (entre 15000 y 30000 hectáreas); y 4) pequeños establecimientos (hasta 15000 hectáreas). 
el resto del país, es decir, que se privilegió al capital antes que al trabajo, ya que el acceso a la tierra fue sencillo, por su abundancia y bajo precio, para aquéllos que podían afrontar los costos de instalación y los gastos de intermediación. Incluso en el extremo austral las condiciones de oferta fueron más benéficas, por tratarse de territorios muy alejados del centro del país, sin comunicaciones y por considerárseles con escasas condiciones naturales para su explotación.

En los inicios de la ocupación las tierras se ofrecieron en arrendamiento con cánones muy bajos, en importantes superficies y con la posibilidad de escoger los terrenos. A los pocos años, los primeros ganaderos instalados se convirtieron en propietarios gracias al dictado de varias leyes que to propiciaron (Burmeister, 1901).

Así, en la primera etapa de la ocupación, que se extiende entre 1880 y 1900 , predominan los latifundios y grandes establecimientos en propiedad y ubicados en los terrenos de mejor calidad y ubicación; en el caso de Santa Cruz, corresponden al sur del territorio. La falta de control estatal en las concesiones dadas por diferentes leyes, los contactos con funcionarios del área y la utilización de terceros para la obtención de lotes, permitieron la concentración.

Los latifundistas reconocen como acumulación previa de capital, el comercio de pieles de guanaco y plumas de avestruz, la caza de anfibios y la búsqueda de oro. Algunos eran empleados de las principales empresas regionales, o bien, se dedicaban a diversas actividades comerciales; gran parte de ellos ya se habían iniciado en la ganadería en Malvinas y sur de Chile. Las mismas sociedades anónimas son las que se instalan en Santa Cruz y Tierra del Fuego y proceden mayoritariamente de Chile, incluso, en el caso del primero de los territorios, los establecimientos se extendían más allá del límite internacional, gozando del beneficio de manejarse con la política nacional que los favoreciera.

Los propietarios de grandes establecimientos también proceden de Malvinas y sur de Chile, varios de ellos se encuentran vinculados con las fuertes empresas regionales, por su participación en calidad de accionistas en distintos negocios (Barbería, 1994).

En la segunda etapa, que se extiende entre 1900 y 1914, se producen modificaciones en la política distributiva, se limitan las posibilidades de acceder a la propiedad, se exige una mayor inversion y aumenta significativamente el canon de arrendamiento. Es así que predomina este último como forma de tenencia, seguido de la ocupación de hecho; y en cuanto al tamaño, sobresalen los medianos y pequeños.

La mayoría de los nuevos ocupantes eran arrendatarios desplazados del sur de Chile al producirse el remate de las tierras que ocupaban, sobresaliendo 
también otros procedentes de Malvinas, que reunían un mínimo capital, que unido a su experiencia en la ganadería ovina regional, les permitía iniciar su propia empresa.

Por último, se distingue una tercera etapa, entre 1914 y 1920 , en la que si bien no se registran cambios en la legislación - salvo la suspensión del derecho a adquirir el cincuenta por ciento de la superficie en propiedad-, en la práctica aparecen trabas para tramitar los contratos de arrendamiento, conduciendo a los interesados a ocupar de hecho los terrenos hasta la firma del mismo, e incluso a su poblamiento sin autorizacion.

De esta manera, la forma de tenencia predominante en la etapa fue la ocupación, en las áreas marginales - teniendo en cuenta la calidad de los terrenos. En cuanto al tamaño, los establecimientos se encuadraban en la categoría de medianos y pequeños.

La procedencia de los pobladores es variada, aún se mantenían los que venían del sur de Chile, pero muchos de ellos, a diferencia de los anteriores, se emplearon en el campo, y luego iniciaron su propio establecimiento. Otros provenían de Buenos Aires, ya sea nacidos alli o inmigrantes.

Las características de los ocupantes es también diferente, ya que no contaron, mayoritariamente, con capital propio ni con experiencia en la actividad ganadera. Muchos de ellos debieron acceder a créditos concedidos por las sociedades anónimas regionales para montar la empresa.

El descenso de la demanda y precios de la lana posteriores a 1918, la reinstalación de los impuestos aduaneros y el aumento de los materiales requeridos para el funcionamiento de los establecimientos encontró a los nuevos productores en proceso de instalacion. El retraso en el pago de las cuotas e intereses de los créditos los obligó a trabajar años para los financistas. Tomando en conjunto los dos territorios, el proceso de ocupación de la tierra entre 1880 y 1920 reconoce el predominio del arrendamiento y la ocupación de hecho; y en cuanto al tamaño de las unidades de producción, sobresalen las medianas y pequeñas.

En cambio, si los consideramos por separado, en Tierra del Fuego prevalecía el latifundio, y en Santa Cruz, los medianos y pequeños establecimientos, si bien, el latifundio y las grandes unidades se ubicaron en las áreas de mejor calidad y ubicación, y se conformaron en la etapa en la que regían condiciones más convenientes para la ocupación.

\section{FORMACIÓN DE UNA REGIÓN ESPECIALIZADA EN LA PRODUCCIÓN OVINA}

La débil presencia estatal, tanto chilena como argentina en la zona sur, facilitó la conformación de una región con autarquía que mantuvo escasas 
relaciones con sus respectivas capitales nacionales. Esta región estaba integrada por el sur de Chile, las Islas Malvinas, Santa Cruz y Tierra del Fuego, el centro de la misma fue la ciudad de Punta Arenas.

La vinculación más estrecha se daba entre los territorios argentinos y chilenos. Aunque Malvinas aportó los primeros capitales y pobladores; un grupo importante de ovejeros y capataces, que se convirtieron en administradores o estancieros en Argentina y Chile; y un modelo de funcionamiento de la empresa ganadera, que se impuso a nivel regional; la fuerte presencia de la Falklands Island Company, empresa londinense que concentraba gran cantidad de terrenos y que, además, funcionaba como intermediaria entre los productores de las islas y los mercados europeos en lo relativo a la venta de lana, manteniéndose al margen del circuito comercial del resto de la región.

La producción de lana y secundariamente de carne, destinada al mercado internacional, fue la especialidad de la región. La carne fue un complemento dirigida, en los inicios, a cubrir la poca demanda local, hasta la instalación de las graserías a principio de siglo y a los pocos años de los frigorificos.

Pero la posibilidad de diversificar la producción sólo pudo ser practicada por los establecimientos cercanos a los frigorificos, dado el elevado costo del traslado de los ovinos. Concretamente, funcionaba uno en Tierra del Fuego y en Santa Cruz tres, pero por su ubicacion, aproximadamente $30 \%$ de las empresas se dedicaban a la producción de carne; incluso en éstos, la lana fue siempre prioridad, Ganadería extensiva, uso intensivo del recurso tierra, escasa mano de obra y mínima tecnologia fueron las características del sistema productivo capitalista implementado.

En toda la región funcionó un mismo modelo de empresa, que sólo difería en relación con el tamaño y con la calidad de las instalaciones. La misma tuvo como objetivo la maximización de beneficios y sus características fueron: la efectivización de una importante y única inversión inicial en infraestrutura; la incorporación de escasa tecnología, sólo bañaderos y molinos de agua; contratación de mano de obra asalariada en forma permanente, hasta las pequeñas estancias contaban con uno o dos peones; y la mínima reinversión de las ganancias en el mejoramiento del establecimiento y en el aumento de la productividad.

Como indicamos, la tierra fue un recurso accesible, tanto por la oferta como por el precio, el problema fundamental para el ganadero fue la obtención de la concesión; y en cuanto a las instalaciones, éstas variaban en relación con el tamaño.

En la instalación de una empresa ganadera regional se cumplían los siguientes pasos: luego de obtenerse la tierra, se compraban los animales 
-que era la inversión más importante por su costo-; luego se levantaban las construcciones mínimas con materiales precarios, consistentes en viviendas e infraestructura para la esquila; en tercer lugar, se alambraba; $\mathrm{y}$, por último, se solucionaba el problema del agua y se edificaban las poblaciones definitivas.

Las instalaciones definitivas consistían en: el alambrado perimetral, que era la inversión más importante por su costo, y las subdivisiones internas. Las construcciones consistían en las viviendas, el galpon de esquila, el bañadero y los corrales; y los molinos con tanques y bebedores o bien pozos con bebedores.

Las viviendas seguían un modelo, también de alcance regional, la más tarde denominada "vivienda patagónica" de origen inglés y adoptada por adaptarse perfectamente al clima muy frío de la zona. Las primeras fueron prefabricadas y se seleccionaban de catálogos que enviaban empresas inglesas; más tarde se construyeron en la región con las modificaciones pertinentes de acuerdo con los diferentes presupuestos. Los materiales utilizados eran madera para la estructura y chapa acanalada para las paredes exteriores y el techo, mientras que en el interior se usaba madera o bien un cartón muy grueso; estas casas resultaban sumamente cálidas, cooperando en ese sentido una muy buena distribución de los calefactores $\mathrm{y}$ una galería vidriada.

El manejo de la hacienda implementado era muy sencillo y suponía escasas actividades, tecnología y mano de obra. Los ovinos permanecían dispersos en el campo la mayor parte del año, alimentándose exclusivamente de pastizales naturales, controlados por peones y ovejeros que recorrían regularmente los terrenos. Las actividades se intensificaban en la primavera, primero con la parición, luego con la señalada, la esquila y, por último, los baños antisárnicos.

En relación con la mano de obra, por las mínimas y simples actividades que suponía un sistema ovino extensivo, la demanda de mano de obra fue muy escasa, como también lo fue el nivel de calificación exigido, sólo los capataces y administradores se buscaban por sus conocimientos en el manejo ovino regional, dado por la práctica.

No obstante, la demanda de mano de obra rural fue la fuente de trabajo más importante de la región, durante la mayor parte del año; la que sólo competía con los frigoríficos durante los meses de faena.

La oferta de mano de obra se componía fundamentalmente de españoles, en especial para las tareas anuales, y de chilenos - procedentes de la isla de Chiloé - para la señalada y la esquila. Estos últimos preferían las actividades estacionales que les permitía regresar a su país o bien a su región hasta la próxima temporada. 
De acuerdo con los datos disponibles, la proporción sería de un trabajador por cada 1200 animales; así, un establecimiento de 10000 hectáreas que por lo general contaba con 2800 ovinos, podía arreglarse con 2 peones estables. Durante la primavera, se incorporaban algunos más para la parición y la señalada; los que también participaban en la esquila, actividad que requería un importante aumento de personal. Finalizada ésta, quedaban unos pocos para los baños. De alli en adelante, sólo los trabajadores permanentes (Morrison, 1917).

Los establecimientos más pequeños contrataban peones y ovejeros. Los que contaban con aproximadamente cuatro o más, incorporaban un capataz y un cocinero; los más grandes, por lo general, sumaban un administrador. Los esquiladores se organizaban en comparsas, que efectuaban la esquila en varias empresas, cobraban por animal. En cuanto a los carreteros, que conducían los fardos de lana desde la estancia a los puertos, cuando realizaban el transporte con carretas que pertenecían a los establecimientos recibian un sueldo mensual; en cambio, los que poseían sus propias carretas cobraban por viaje.

La comercialización de la producción se efectuaba en forma individual y en consignación, los ganaderos trasladaban la lana del establecimiento a los puertos, y desde alli, la conducían al de Punta Arenas, siendo en éste transbordadas a los buques internacionales con destino a Londres, Hamburgo y Amberes. En estos puertos se efectuaban los remates de fardos; una vez vendidos, las casas consignatarias giraban los importes correspondientes vía los bancos de la ciudad de Punta Arenas. Más adelante estas casas se instalan en la región, lo cual favoreció a los ganaderos ya que se evitaron una parte de la intermediación y les posibilitó un conocimiento más certero de la suerte que correría la producción.

\section{Funcionamiento de la región}

Como indicamos con anterioridad, a la introducción del ovino, Punta Arenas no sólo fue el único núcleo urbano de la Patagonia Austral, sino que ya se vislumbraba el rol que ocuparía en la zona. Si bien Chile no puso mayor énfasis en su política de poblamiento de los territorios del sur, fue mucho más efectiva que la implementada por la Argentina. Las medidas adoptadas para el asentamiento de inmigrantes y la utilización de su puerto como punto de recalada y aprovisionamiento del transporte marítimo internacional, le dieron gran impulso.

Los primeros pobladores tentaron todas las vías posibles de explotación que la zona ofertaba, sin respetar los cuestionados límites entre ambos países. Así es que los futuros territorios de Santa Cruz y Tierra del Fuego 
fueron permanentemente recorridos, ya sea para comerciar con los indígenas, para buscar oro, para cazar lobos marinos o para el salvataje de mercaderias náufragas.

A partir de 1885 se produce la migración masiva de pobladores de Chile, que se instalan en los territorios argentinos como ganaderos o mano de obra rural. El avance de la frontera ovina generó necesidades que también fueron cubiertas por la ciudad de Punta Arenas y áreas circundantes. De sus aserraderos fueron las maderas que se utilizaron en la construcción, las que transportaron - al igual que las ovejas - en buques fabricados y/o reparados en sus astilleros; como también las casas comerciales y bancarias instaladas en Santa Cruz, fueron sucursales de las chilenas.

En la figura 1 se pueden apreciar las áreas de Santa Cruz y Tierra del Fuego ocupadas con establecimientos ganaderos formados por pobladores procedentes de Chile. También se incluyen los centros urbanos que contaron con sucursales de casas comerciales con sede en Punta Arenas.

La más importante de las empresas fue la Sociedad Anónima Importadora y Exportadora de la Patagonia, constituida en 1908, de la fusión de dos firmas; José Menéndez, con sucursales en Río Gallegos y Puerto Santa Cruz; y Braun y Blanchard, con casas en Río Gallegos, en Puerto Santa Cruz y Puerto San Julián.

Esta compañía monopolizaba el comercio de importación y exportación; el transporte regional, actuaba como representante de agencias internacionales de navegación; y otorgaba créditos. Contó con una flota mercante propia, que primero se encargó del cabotaje y la conexión con las líneas europeas que transportaban la producción; $y$, más tarde, hizo lo propio con la relación entre los puertos de la zona y el de Buenos Aires.

Esta empresa tenía sucursales en Río Grande, en todos los puertos y pueblos del interior de Santa Cruz, incluso en el territorio de Chubut. Todos los pobladores tenían vinculacion con la comúnmente denominada " $\mathrm{La}$ Anónima"; es decir, que desde principio de siglo sustituyó las compras que los ganaderos efectuaban anualmente, a través de catálogos, en las casas europeas.

Otras firmas comerciales cuyas sedes se encontraban en Punta Arenas fueron: la Compañía Argentina del Sud, que surge de la fusión de tres comercios que ya funcionaban en Santa Cruz, ellas fueron Muller Hnos. —en Puerto San Julián y Bahía Laura-, Stubenrauch y Cía. —en Puerto Deseado y Las Heras y Federico Brhome- en Río Gallegos; Lennox Dobrée y Cía.; Rivera e Iglesias y Maximiliano Gilli. (Martinic, 1976).

$\mathrm{El}$ avance de estas empresas impidieron el surgimiento del comercio local, si bien en Santa Cruz lograron prosperar algunos, fruto de la 
asociación de conocidos ganaderos. La más importante fue la Sociedad Anónima Mercantil de la Patagonia, formada en 1918, con sucursales en Rfo Gallegos, Puerto Santa Cruz, Puerto Coyle, Piedra Buena, Puerto Deseado, Las Heras y Pico Truncado; dedicada al comercio de ramos generales, a la exportación e importación, créditos, agente de compañías de vapores y representante de casas compradoras de lana y frutos del país. Otra fue la firma conocida como Varela y Femández, que solo funcionaba en Río Gallegos; y la Sociedad Anónima Industrial y Comercial de San Julián instalada en el pueblo del mismo nombre (Correa, 1979).

En cuanto a los bancos, en Santa Cruz funcionaban dos con sede en Punta Arenas; el de Tarapacá y Londres, desde 1899 en Río Gallegos, y más adelante con sucursales en Puerto San Julián y Puerto Deseado; y el de Punta Arenas-Magallanes, que inició sus actividades en 1912 en Puerto Santa Cruz, en 1916 en Puerto San Julián, y en 1918 en Río Gallegos (Correa, 1979).

También el Banco de la Nación Argentina tenía una sucursal en Río Gallegos, pero no podía competir con los anteriores, por las dificultades de los pobladores del interior para efectuar trámites en la capital del territorio; además los compradores de las lanas de la región giraban los importes desde Europa a través de los bancos chilenos.

En el transporte y comercialización de la lana también Punta Arenas jugó un rol fundamental, ya que, por lo general, los fardos de lana se entregaban en consignación a compañías que los remataban en Londres, Hamburgo y Amberes, que como indicamos, giraban los importes a través de los bancos de Punta Arenas, más tarde con sucursales en los puertos santacruceños.

La industria frigorifica también se inició en Chile, con la apertura del frigorífico de "Río Seco" en 1905 en Punta Arenas, al que siguio "Puerto Sara" en 1908 en San Gregorio, "Puerto Bories" en 1912 en Ultima Esperanza, "Punta Arenas" y "Puerto Natales" en 1918 (Martinic, 1976).

En Santa Cruz funcionaron tres, abiertos en la primera década del presente siglo: Swift en Río Gallegos y San Julián, y Armour en Santa Cruz. Después de 1920, se habilitó otro en Puerto Deseado, a cargo de una sociedad de ganaderos, encabezados por Alejandro Menéndez Behety y Mauricio Braun. Mientras que en Tierra del Fuego, en 1917 se habilitó uno en Río Grande, propiedad del grupo Braun-Menéndez con la participación de otros productores.

A pesar del funcionamiento de los frigoríficos en los territorios argentinos, gran parte de los ovinos continuaron vendiéndose a los frigoríficos chilenos. En el caso de Puerto liatales, por su cercanía a la zona de los lagos del sudoeste de Santa Cruz ya que el área de influencia de este centro 
alcanzaba hasta los lagos San Martín y Pueyrredón; en los restantes, influyó directamente la participación en las acciones de los capitalistas regionales que poseían estancias en el territorio (ver figura 1 ).

A modo de ejemplo, de la revisión del listado de socios de la Sociedad Anónima Frigorífico Puerto Natales, resulta que veintidós de ellos eran ganaderos de Santa Cruz y sur de Chile (Archivo Instituto de la Patagonia, 1917). Por otra parte, las empresas chilenas mantuvieron una politica de incentivo a la producción, mediante la fijación de precios adicionales a los que obtuvieran mayor peso por animal.

En cuanto al transporte marítimo, desde el inicio de la ocupación de los territorios argentinos las empresas instaladas en Punta Arenas to monopolizaron. Primero se encargaron del traslado de los pobladores, de los ovinos y de los materiales para las construcciones rurales; y luego, del aprovisionamiento a las estancias y concentraciones urbanas, y de la conducción de la producción lanera desde los puertos a Punta Arenas. Ya en 1893 navegaba el vapor "Amadeo" de José Menéndez, y los barcos de la sociedad Braun y Blanchard llegaban hasta Puerto Deseado.

Allí se transbordaban a los buques de alguna de las ocho empresas europeas de navegación. Ya en 1898, seis compañías internacionales de navegación contaban con agencias en esa ciudad: Pacific Steam Navigation Company, con dos buques por mes; Lloyd Norte Aleman, uno semanal; Messageries Maritimes, uno quincenal; Kosmos, quincenal (una vez por mes toca las Malvinas); Chargeurs Réunis, quincenal; una compañía italiana, cada veinte días y una norteamericana, en forma irregular. Y funcionaban cuatro locales: Braun y Blanchard, con cuatro buques; Curtze y Wahlen, con dos; y "..,la Sociedad Anónima que arma el Albatros..." (Martinic, 1976).

Los territorios argentinos no contaban con otros medios de comunicación marítima, ya que el gobierno nacional, fiel a su política liberal, la dejo en manos de los capitalistas regionales, y ni siquiera intento el establecimiento de una línea regular que acercara estas tierras al resto del país. Fueron constantes las solicitudes de los gobernadores para contar con una línea regular hasta Buenos Aires, debido a que los transportes nacionales realizaban uno o dos viajes al año, además de no contar con capacidad, demoraban sesenta díaš entre ida y vuelta.

En cuanto a la red de caminos, en el caso de Santa Cruz (como se aprecia en la figura 1) existía uno principal que unía los puertos con los centros poblados ubicados en la meseta y en la cordillera, siguiendo el curso de los ríos; desde las estancias principales se abrían algunos que entroncaban con éstos. Son notables los que corresponden al sur, especialmente los que finalizan en Puerto Natales y Punta Arenas. Esta direccion 
de los caminos muestra claramente la calidad de tributario de este territorio en relación con esos centros.

Como se aprecia en la figura 1, el área de influencia de Puerto Natales alcanza a la zona de los lagos del sur, que incluye el traslado de lana, de ovinos para sus frigoríficos, de mercaderías y de mano de obra. Mientras que la expansión de la hegemonía puntarenense cubre todo el territorio y en especial el sur, donde las estancias continúan más allá del límite internacional, y efectúan todas sus operaciones directamente en esa ciudad.

La expansión puntarenense llegó a la educación, gran parte de los niños argentinos estudiaron alli, antes y después de la creación de escuelas en los territorios, como también otros recibieron su instrucción en colegios de Londres, producto de la idiosincracia inglesa que tendió a la defensa y perduración de sus costumbres; afectó también el campo de la salud, muchos pobladores contaban con un certificado de nacimiento originado en Punta Arenas, evidentemente aquél que tenía la posibilidad, prefería un centro con mayores garantías que pueblitos con uno o ningún médico. También había dependencia religiosa, ya que los Salesianos de Santa Cruz y Tierra del Fuego pertenecían a la Prefectura Apostólica de la Patagonia Meridional y Tierra del Fuego, creada en 1883 con sede en Punta Arenas.

Esta influencia magallánica, si bien se extendio a todo el territorio, fue más fuerte en el sur de Santa Cruz y en el norte de Tierra del Fuego, las razones son por demás elocuentes: fue la zona inicial de la ocupación y la preferida por su calidad; y en el caso de Santa Cruz, por la posibilidad de reunir unidades de producción que se extendieran a ambos países, que no sólo permitía la formación de latifundios, sino también la de elegir la política más conveniente.

Queda claro con lo expuesto la formación de esta región autárquica, integrada por el sur de Chile, Santa Cruz y Tierra del Fuego, basada en la producción y exportación de lana y carne a los mercados europeos. Como también que el centro regional fue la ciudad de Punta Arenas, primer establecimiento, y desde el cual se iniciaron las diversas actividades económicas de las áreas circundantes, que dieron vida a Magallanes y permitieron la acumulación de capital, que en los últimos años del siglo, se invirtio en la ganadería ovina, expandiéndose también en territorio argentino (ver figura 1).

De esta manera, Santa Cruz y Tierra del Fuego se constituyeron en un área periférica del sur chileno, en razón de que así como los capitales que dieron comienzo a la ocupación se originaron alli, también los ingresos que generaron se dirigieron a Punta Arenas; salvo las inversiones requeridas para el funcionamiento de los establecimientos ganaderos y de los edificios de las casas comerciales y bancarias, el resto fue girado a esa 
ciudad, y más tarde a otros puntos. Al respecto, el gobernador de Santa Cruz, Dutari Rodríguez, manifiesta

...Pudimos evitar que Chile se hiciera dueño de estos ricos territorios; pero no hemos echado afuera su espíritu y su influencia, que imperan en la mitad de la Patagonia Argentina. Todo el comercio de Santa Cruz, rico y poderoso, radica en Punta Arenas, y las lanas de este territorio, que son de calidad superior, se cotizan en los mercados europeos con el nombre de esta ciudad... Punta Arenas es un ejemplo, es ya un centro de movimiento asombroso, una linda ciudad... Pero Chile la cuida como un tesoro; ...

...Yo pienso que no se puede dejar librados a sus propios impulsos al pueblo de Gallegos, que tiene que ser y debe ser la metrópoli de estos inmensos territorios del Sud. Yo pienso que debemos traer aquí el alma argentina, $\mathrm{y}$ para conseguirlo son indispensables las comunicaciones rápidas y frecuentes con esta Capital. Actualmente, las comunicaciones de Buenos Aires, que pudieran llegar en ocho días por vía del Estrecho, demoran un mes, por lo regular, y son traídas en vapores subvencionados por el gobierno de Chile... (Archivo General de la Nación, 1906).

\section{El rol de los grandes empresarios en la conformación y funcionamiento de la región}

La ciudad de Punta Arenas fue en realidad beneficiaria indirecta del proceso de ocupación de los territorios argentinos, ya que los verdaderos protagonistas en la conformación y funcionamiento de la región fueron los grandes empresarios - de Malvinas y sur de Chile-, los que luego de su consolidación, buscaron nuevos horizontes de inversión, y vieron en los territorios de Tierra del Fuego y Santa Cruz un campo propicio, ya sea para la expansión ganadera, como para la ampliación de mercados, para sus negocios comerciales, bancarios y de transporte. Como también, cuando las posibilidades de crecimiento regional se detengan, invertirán en otras zonas de Chile y Argentina.

Por supuesto que no debemos olvidar que a Chile le convenía la presencia de los que consideraba empresarios chilenos en territorio argentino, y que aprovechaba las posibilidades que el funcionamiento regional le permitía para fortalecer su influencia. Situación que denuncian permanentemente los gobemadores. Escribe el gobernador Dutari Rodríguez al Ministerio del Interior, en 1906,

...El gobiemo de Chile paga una subvención anual de veinte mil pesos a la Cía. de vapores Braun y Blanchard por el servicio de correspondencia 
entre Gallegos, otros puntos del territorio argentino y Punta Arenas; pero a designio de este servicio se hace en combinación con los paquetes que vienen del Pacífico y no con los de Buenos Aires...

Así resulta que la prensa de Santiago y Valparaíso puede llegarnos aquí en ocho días, mientras demoran un mes largo los diarios argentinos, que podrían venir fácilmente por vía Punta Arenas, en igual o más breve periodo de tiempo. Con frecuencia sucede el caso de coincidir las fechas de llegada de los paquetes de Buenos Aires y Montevideo - Punta Arenas, con la salida de los vapores de Braun y Blanchard para este puerto, Santa Cruz y San Julián; pero esta salida siempre se hace anticipándose en una o dos horas a aquella, como para no traer a estas regiones correspondencia argentina...

...De modo que Chile hace imperar aquí combinaciones postales que paga a buen precio, calculadas para su provecho propio... tiene monopolizado como riquísimo mercado para sus productos todo el extenso territorio de Santa Cruz... Aquí no se conoce nada argentino, y sin embargo, Valparaíso no dísta de Gallegos menos que de Buenos Aires... (Archivo General de la Nación, 1906).

La formación de los capitales regionales reconoce un primer momento, basado en la explotación de recursos destinados a la exportación: caza de lobos marinos, comercialización con los indígenas de pieles de guanaco y plumas de avestruz — como también su caza directa-; y además la minería aurifera.

En un segundo momento, a partir de 1880 aproximadamente, se incorpora la ganadería ovina, circunscrita a las áreas cercanas a Punta Arenas, luego extendida al resto de los terrenos de Magallanes y Última Esperanza (Chile), y simultáneamente a Tierra del Fuego y Santa Cruz. Estas actividades generaron el desarrollo de otras: comercios, bancos, frigorificos y aserraderos; en todas ellas estuvieron presentes los principales capitalistas, ya sea iniciándolas y/o monopolizándolas.

En los cuadros 1 y 2, presentamos, a modo de ejemplo, los emprendimientos de los dos grupos más poderosos e influyentes en la región, como son las familias Menéndez Behety y Braun; apreciamos la variedad de actividades que cubrían, las que abarcaban tanto la ganadería, como los rubros industrial, comercial y financiero. Como vemos, la evolución de sus negocios es también la de la región. Así, luego de las primeras actividades que permitieron la acumulación del capital inicial, se dedicaron tanto a la explotación ovina, como a los servicios y otras actividades, que la misma generó. Si bien contaron con enormes extensiones, tanto en Argentina como en Chile, ubicadas en los mejores terrenos; quizás, en cuestión de ingresos, fueron más significativos los negocios relacionados con el comercio, el transporte y las finanzas. 
Cuadro 1. Grupo Menéndez Behety (ganadero, comercial, industrial y financiero).

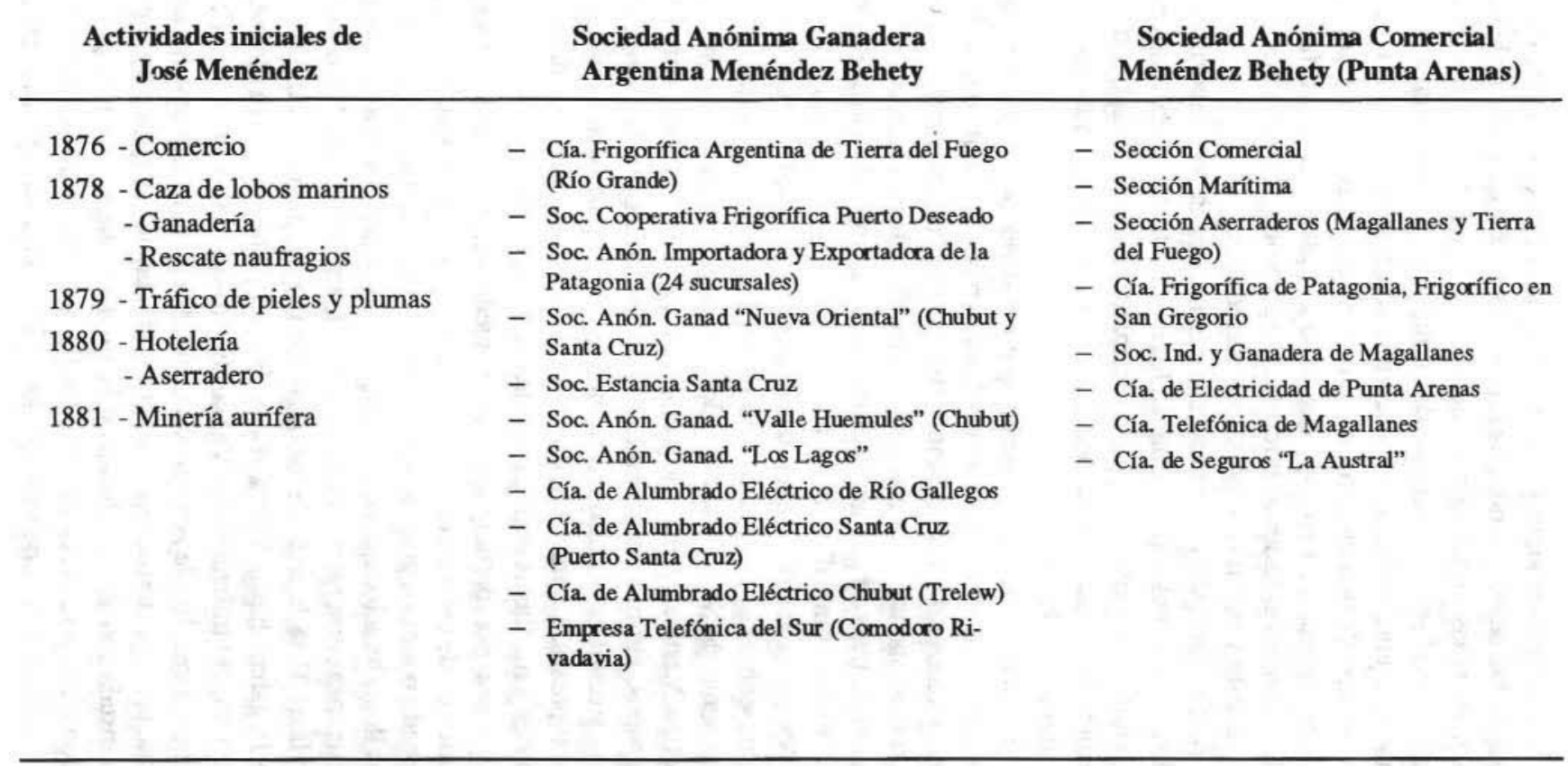

FUENTE: Martinic Beros (s/f.). “Actividades iniciales de José Menéndez", Revista Argentina Austral. 
A principio de siglo ya monopolizaban el comercio y el transporte, a través de la Sociedad Anónima Importadora y Exportadora de la Patagonia, con numerosas sucursales en el territorio, que podemos apreciar en la figura 1, y el servicio regular de comunicación maritima. Esta sociedad también se encargaba de la comercialización de la lana, de la importación de todo tipo de mercaderías, de la conexión con agencias internacionales de navegación; y, también, concedía créditos. La mayoría de los establecimientos ganaderos, iniciados después de 1914, contaron con préstamos de ésta y de otras importantes sociedades.

Fueron también los principales accionistas de los frigoríficos del sur chileno; a los cuales se destinaban los ovinos de sus establecimientos, y de los numerosos ganaderos que participaban de la sociedad, o que por una cuestión de distancia y de beneficios, optaban por vender los animales a los frigorícos chilenos.

Mauricio Braun participaba - en calidad de socio- en entidades bancarias, que tenían su sede en Punta Arenas, y sucursales en los principales núcleos urbanos de Santa Cruz, los que concentraban las transacciones relacionadas con la venta de la lana. Por último, intervinieron en otras empresas, tales como las compañías de alumbrado eléctrico, empresas telefónicas, compañías de seguros, etcétera.

Aparte de las relaciones familiares que se entablaron entre estos dos grupos entre si, y con otros de la región - los más notables fueron el casamiento de Sara Braun con José Noqueira, el de Mauricio Braun con Josefina Menéndez Behety, y el de dos hijas de Mauricio con integrantes de grupos, también importantes en la región, como son Campos y Caminos-su estrategia consistio en incorporar numerosos accionistas en todas sus empresas. La mayoría de los ganaderos con unidades de producción grandes y medianas eran socios de estas empresas.

Ya sea por participar en las sociedades, por actuar en calidad de testaferros de establecimientos en Santa Cruz, o por contar con un crédito otorgado por estos grupos, la mayoría de los ganaderos estaban vinculados con ellos; situación que se extiende al resto de la población que se abasteció en sus casas comerciales y compañías de transporte (Fish y Braun, 1931).

Hasta 1918 la sede de los negocios fue la ciudad de Punta Arenas, los que llegaban hasta Valparaíso y Buenos Aires; a partir de ese año se dividirán, centralizando en Valparaíso, los correspondientes a Chile, y en Buenos Aires, los de Argentina. La modificación de la coyuntura internacional, la ocupación de prácticamente todas las tierras aptas para la ganadería, la reinstalación de la aduana, y la implementación de una política más nacionalista, son las causas que los llevaron hacia horizontes más beneficiosos para sus inversiones, que la región ya no ofrecía (Morrison, 1917). 
En Argentina, el grupo Braun-Menéndez Behety se concentró en la Capital Federal, incursionando en empresas tales como Astilleros Río de La Plata, ASTARSA (construcciones navales, ferroviarias y viales), en compañías de seguros, financieras, inmobiliarias, y en otras relacionadas con el turismo, con maquinarias agrícolas y asistencia médica, controlando también la editorial Emecé.

Es decir, que estos grupos no se especializaron en la ganadería, ni tampoco fue su actividad fundamental, ya que sistemáticamente, fueron reinvirtiendo sus ganancias en nuevos rubros, que el crecimiento ganadero requería. Por lo tanto, se convirtieron en los principales receptores de los ingresos que la producción ovina generaba a nivel regional. Al reducirse las expectativas de desarrollo en el área sus inversiones se dirigieron a otras regiones argentinas y chilenas.

\section{LA DESINTEGRACIÓN DE LA REGIÓN}

La desintegración de la región, deviene de varios factores que se modificaron entre 1914 y 1920, y que dieron fin a la coyuntura muy favorable que caracterizamos en los años previos. Estos factores se dieron tanto a nivel internacional, como nacional y regional.

La apertura del Canal de Panamá, en 1914, restó importancía al Estrecho de Magallanes en la navegación mundial, interfiriendo en la comunicación directa que la región mantenía con los mercados europeos. Lentamente, a partir de 1914, en el caso de los territorios argentinos la producción de lana se fue exportando a través del puerto de Buenos Aires.

La Sociedad Anónima Importadora y Exportadora de la Patagonia monopolizo la vinculación de la región con el puerto de Buenos Aires, (ver figura 1), a su vez, contaba con una línea de vapores, cuyo itinerario fijo contemplaba todos los puertos de Tierra del Fuego y Santa Cruz, tocando también los del resto de Patagonia.

El descenso de la demanda y precio de la lana, que de $\$ 30$ los $10 \mathrm{~kg}$ que se pagaba durante la guerra, baja a \$8 a partir de 1918; ascendiendo recién a partir de 1923, pero sin alcanzar el valor máximo de la etapa.

La crisis se prolongó durante años, para los establecimientos iniciados con posterioridad a 1914, que contaron con créditos para su instalación y no pudieron hacer frente al pago de las cuotas, debiendo convenir nuevas formas de cancelación que los obligó a entregar parte de su producción.

Por último, incide especialmente la política de integración territorial encarada por ambos países; fundamentalmente la reimplantación de los impuestos aduaneros, en 1912 en Chile y en 1918 en Argentina, que afectó la rentabilidad de las empresas ganaderas. Especialmente se vieron más 
Cuadro 2. Grupo Braun (ganadero, comercial, industrial y financiero).

\section{Actividades iniciales de Mauricio Braun}

- Empleado de José Noguerira

- Actividades relacionadas con la caza de lobos marinos

-1880 , transporte de ovinos

\section{Área marítima}

- 1884 Soc. Braun-Scott (cabotaje regional)

- 1892 Braun-Blanchard (cabotaje, astilleros, agencia Cías. de ultramar)

- 1907 Empresa naviera (toda la costa chilena)

- 1929 Cía. Chilena de Navegación Interoceánica

\section{Frigoríficos}

Participación en: Río Seco

Rio Grande

\section{Puerto Deseado \\ Puerto Sara \\ Puerto Bories \\ Puerto Natales}

\section{Área comercial}

-1892 Braun y Blanchard

- 1908 Soc. Anón. Importadora y Exportadora de la Patagonia

\section{Otras empresas}

- 1897 Cía. de Electricidad de Punta Arenas

Empresa Telefónica San Julián

- 1904 Soc. Ballenera de Magallanes Cía. Minera Cutter Cove

- 1900 Banco de Punta Arenas (Chile y Argentina)

- 1915 Cía. de Seguros la Austral

- 1918 Cía. Telefónica de Magallanes

\section{Área ganadera}

- Soc. Ganad. Argentina

- Soc. Pastoril Glencross

- Sindicato Santa Cruz

- Soc. Estancia Santa Cruz

- Southern Patagonia Sheep Farming Company

- Cía. Explotadora Cerro Palique

- Soc. Explotadora de Tierra del Fuego

- Sindicato San Julián

- Soc. Ind. de Puerto Aysen

- The San Julián Sheep Farming Company

- Soc. Estancias Lago Posadas

- Soc. Ganad. Valle Huemules

- Soc. Estancia Los Manantiales.
Estancias en Santa Cruz: Coy Aike

Monte Negro

Los Machos

Tcheon-Aike

Sofia

Cerro Comisión

Participación en las

estancias: La Maciega

Tapi-Aike

María Inés

San Elías

Vidalita

Cancha Rayada

La Federica

El Librún

La Josefina

Coluhuel-Aike

San Mauricio

La Flora

La Carlota.

\section{Estancias en Chubut: Quichaura}

Pepita

Fuente: Martinic Beros (s/f), Revista Argentina Austral. 
perjudicadas las que se encontraban en proceso de instalacion, por el encarecimiento de los materiales requeridos para montar la infraestructura.

Otras medidas del gobierno argentino tendientes a la integración nacional de los territorios del sur que afectaron la conformación de la región, son: la obligación para las empresas que actuaban en la zona de contar con sede en la capital federal, es asf que a partir de 1918, los negocios que la Sociedad Anónima Importadora y Exportadora de la Patagonia se centralizaron en la ciudad de Buenos Aires; y la Ley de Cabotaje Regional.

La Ley de Cabotaje Nacional, del 1 de enero de 1912, determina:

... se concederá a los buques de las naciones limítrofes el mismo tratamiento que a los buques nacionales de cabotaje, siempre que dichas naciones concedan a los buques argentinos, a título de reciprocidad, el mismo tratamiento y franquicias que a sus propios buques...;

aclarando que el cabotaje se reserva para la Marina Argentina. Los que no se incluyeran en lo estipulado, deberían abonar en cada viaje, como derecho de fondeadero, $\$ 100$ moneda legal argentina, si fueran considerados como vapores de correo, para los restantes casos, regiría doble tarifa.

Evidentemente, lo establecido perjudicaba directamente a las compañías chilenas, ya que todo el cabotaje regional se realizaba en embarcaciones de esa procedencia; los que tocaban todos los puertos de Santa Cruz, Chubut y Tierra del Fuego; mientras que la presencia de buques argentinos en Punta Arenas era nula.

Pero las empresas chilenas continuaron monopolizando el cabotaje regional, utilizando para ello el cambio de bandera. Esta actitud es corroborada por el diputado chileno Agustín Gómez García,

...la nueva ley argentina viene a perjudicar a los armadores de Magallanes, que no pueden hacer operaciones de cabotaje en la costa argentina sino cambiando la bandera, como ya lo han hecho... conviene que nuestro Gobiemo negocie con el Plenipotenciario argentino el establecimiento de la reciprocidad entre ambos países, en la cuestión del comercio marítimo de cabotaje.

... Además de la tarifa aludida - ...de cinco a seis mil pesos moneda argentina al año... - existe la prohibición a estas naves de hacer operaciones de transporte entre uno y otro puerto de la referida costa. Asimismo, debe señalarse un peligro más para nuestra Marina Mercante: el gobierno argentino, basado en la nueva ley de cabotaje, que dispone de que los buques de ultramar, sólo podrán hacer operaciones en los puertos mayores de la República, declarando que no bay ningún puerto mayor desde Madryn al sur, con cuya medida ha excluido del todo la bandera 
chilena en esa costa, y por consiguiente, la desaparición de nuestra flota mercante nacional; asestó un golpe de muerte a la actividad comercial e industrial de Magallanes... (Gómez, 1914).

La ley núm. 10606, de 1918, profundiza la medida, ya que además de reservar el comercio de cabotaje para buques de bandera nacional, determina que para ser considerados como tales deberán: usar el pabellon argentino, contar con capitanes argentinos o nacionalizados y con la cuarta parte de la tripulación argentina. La ley acuerda la devolución, a los armadores que construyeran buques en el país, de los derechos aduaneros abonados por los materiales introducidos para la construcción de los mismos, siempre que no produzcan en el país en cantidad y calidad suficientes. Esta ley coincidirá con dos medidas que agravarán la situación de las compañías de navegación y de comercio puntarenense. Que son; a) los derechos que, por disposición del gobierno chileno, deberán abonar todos los buques que recalen en ese puerto, tanto para el traslado de pasajeros como de mercaderías, y $b$ ) la decisión del mismo gobierno de reimplantar la aduana en Magallanes (Gomez, 1914).

Al respecto, el gobiemo de Magallanes escribió al ministro de Hacienda

... fácil es comprender que, dadas las enormes riquezas y gran extensión territorial de la República Argentina, le sèrá fácil a esa nación subsanar los inconvenientes de algunos de sus puertos de la Patagonia, que no permite la entrada de vapores de gran calado, y entonces, bajo el régimen de franquicia aduanera, le será muy fácil quitar a Punta Arenas su hegemonía comercial, hegemonía que, como ya lo he dicho, se va perdiendo, pues es sabido que a la región chilena de Ultima Esperanza se llevan mercaderías de Río Gallegos. También es público y notorio que varias sociedades comerciales como la de Menéndez Behety, ha lanzado al comercio vapores con bandera argentina en vez de chilena, debido a la terminación del régimen franco... (Martinic, 1976).

El traslado de la sede de los negocios de Braun y Menéndez Behety a la capital federal, en 1918, y a Valparaíso, para las actividades correspondientes al sur chileno; $y$ especialmente las nuevas inversiones efectuadas en la provincia de Buenos Aires, y en el centro de Chile, nos hablan del fin de la región autárquica y del cierre de la coyuntura tan favorable para la producción lanera que vivió la zona hasta 1920. Por otra parte, la ocupación de las mejores tierras y del mantenimiento de un sistema ovino extensivo, limitaban la expansión de los capitales de las mencionadas empresas. También en 1923 se levantan las sucursales que el Banco de Punta Arenas tenía en el territorio de Santa Cruz. 
Queda claro con lo expuesto, la formación de una región autárquica, integrada por el sur de Chile, Santa Cruz y Tierra del Fuego, basada en la producción y exportación de lana y carne a los mercados europeos; con centro en la ciudad de Punta Arenas, primer asentamiento, y desde el que se iniciaron las diversas actividades económicas de las áreas circundantes, que permitieron la acumulación de capital, que en los últimos años del siglo, se invirtieron en la ganadería ovina. En este contexto, los territorios de Santa Cruz y Tierra del Fuego se constituyeron más que en un área marginal de la Argentina, en periferia regional, por lo menos hasta 1920.

\section{BIBLIOGRAFIA}

Archivo Instituto de la Patagonia. 1917. Registro de la Propiedad, Punta Arenas, núm. 33.

Archivo General de la Nación, 1906. Fondo Documental Ministerio del Interior, expediente núm. 660.

BARBERIA, Elsa Mabel.1993. "Chile y Argentina. Una región autárquica en el sur, 1880-1920", Revista Waxen, núm. 4, Universidad Federal de la Patagonia Austral, Río Gallegos.

- 1994. "El proceso de ocupación de la tierra en el territorio de Santa Cruz, 1880-1920", inédito, 1994.

BURMEISTER, Carlos. 1901. Memoria sobre el territorio de Santa Cruz, Memoria del Ministerio de Agricultura, Imprenta de la Nación, Bs. As. Argentina.

CORREA Falcón, Edelmiro y Luis Klappenbach. 1979. La Patagonia argentina, Bs. As.

FISH y Braun Menéndez, 1931. El problema de la tierra pública en la Patagonia. La sociedad anónima Estancias Mauricio Braun ante la Dirección General de Tierras y Colonias, Bs. As. Argentina.

GOMEZ García, Agustín. 1914. "Viaje de un chileno a Magallanes en 1914". El Mercurio, 15 de abril.

ISOLA, Viviana. s/f. "Las relaciones económicas de la Patagonia Austral Argentina - Santa Cruz y Tierra del Fuego- con Punta Arenas en el periodo 1885-1914", (mimeo).

LAFUENTE, Horacio. 1981. La región de los Césares. Apuntes para una historia económica de Santa Cruz, Edit. de Belgrano, Bs. As.

MARTINIC Beros, Mateo. s/f. "Actividades iniciales de José Menéndez". Revista Argentina Austral.

- 1972. Magallanes, síntesis de tierra y gentes, Edit. Francisco de Aguirre, Bs. As. 
- 1976. La expansión económica de Punta Arenas sobre los territorios argentinos de la Patagonia y Tierra del Fuego, 1885-1925, Anales Instituto de la Patagonia, Punta Arenas.

MORRISON, Jorge. 1917. La ganadería en las regiones de las mesetas australes del territorio de Santa Cruz, Imprenta de Luis Vaggia, Bs, As.

SAROBE, José María. 1935, La Patagonia y sus problemas. Estudio geográfico, económico, político y social de los territorios del sur, Aniceto Lopez Edit., Bs. As. Argentina. 